\title{
Klebsiella pneumoniae infection associated septic pulmonary embolism in an emergency department from east China
}

\author{
Xiaobin Zhang ${ }^{1 \#}$, Qian Yang ${ }^{1 \#}$, Bo Gao ${ }^{1,2}$, Jialu Wang ${ }^{1}$, Lei Tian ${ }^{1}$, Jia Hua ${ }^{3}$, Changqing Zhu ${ }^{1}$, Xiaoye Lu ${ }^{1}$ \\ ${ }^{1}$ Department of Emergency Medicine, Ren Ji Hospital, School of Medicine, Shanghai Jiao Tong University, Shanghai, China; ${ }^{2}$ Department of \\ Critical Care Medicine, Pu Dong Hospital, Shanghai Fudan University, Shanghai, China; ${ }^{3}$ Department of Radiology, Ren Ji Hospital, School of \\ Medicine, Shanghai Jiao Tong University, Shanghai, China \\ Contributions: (I) Conception and design: X Lu, C Zhu; (II) Administrative support: Ren Ji Hospital, Science and Technology Commission of \\ Shanghai Municipality; (III) Provision of study materials or patients: X Zhang, Q Yang, B Gao, L Tian; (IV) Collection and assembly of data: X \\ Zhang, Q Yang, J Wang; (V) Data analysis and interpretation: X Zhang, Q Yang, J Hua; (VI) Manuscript writing: All authors; (VII) Final approval of \\ manuscript: All authors. \\ "The authors contributed equally to this work. \\ Correspondence to: Dr. Xiaoye Lu. Associate Chief Physician, Emergency Department, Ren Ji Hospital, School of Medicine, Shanghai Jiao Tong \\ University, Shanghai 200127, China. Email: luxiaoye_rj@163.com.
}

Background Septic pulmonary embolism (SPE) is attracting more attention as a special pulmonary sign in severe infection. We aimed to describe the clinical and imaging features of Klebsiella pneumoniae (K. pneumonia)-associated SPE in the emergency department.

Methods: Records of patients with primarily extrapulmonary infection of $K$. pneumoniae who were admitted to the emergency department between 2014 and 2019 were retrieved. The identifications of K. pneumoniaeassociated SPE were mainly dependent on the clinical manifestations, typical imaging findings, and presence of a primary source of $K$. pneumoniae infection.

Results: A total of 33 cases were identified as SPE with extrapulmonary $K$. pneumoniae infection. The main clinical manifestations were a febrile/fragile state (100\%), respiratory symptoms $(18.2 \%)$, and digestive symptoms (33.3\%). Eight patients (24.2\%) developed septic shock, 2 (6.0\%) experienced respiratory failure, and $2(6.0 \%)$ complicated endophthalmitis. The major source of infection was liver abscess $(\mathrm{n}=26,78.8 \%)$, followed by septicemia $(n=8,24.2 \%)$, intestinal infection $(n=3,9.1 \%)$, and ascites $(n=1,3.0 \%)$. The computed tomography (CT) features included the following: peripheral wedge-shaped opacity $(\mathrm{n}=12,36.4 \%)$, a feeding vessel sign ( $\mathrm{n}=3,9.1 \%)$, multiple nodular lesions $(\mathrm{n}=5,15.2 \%)$, multifocal lung ill-infiltrations $(\mathrm{n}=15,45.5 \%)$, patchy ground-glass opacities $(n=6,18.2 \%)$, focal consolidations $(n=9,27.3 \%)$, lung abscesses $(n=4,12.1 \%)$, and pleural effusion $(\mathrm{n}=21,63.6 \%)$. Re-examination of lung HRCT conducted in 7 patients demonstrated imaging improvement after treatment.

Conclusions: K. pneumonia-SPE presented special clinical and imaging characteristics, which bear similarities to the signs of pneumonia, but was potentially catastrophic. Identifying SPE in septic conditions is crucial to improving clinical outcomes.

Keywords: Septic pulmonary embolism (SPE); Klebsiella Pneumoniae; imaging findings

Submitted Dec 22, 2019. Accepted for publication Sep 24, 2020.

doi: 10.21037/apm-19-648

View this article at: http://dx.doi.org/10.21037/apm-19-648 


\section{Introduction}

Septic pulmonary embolism (SPE) is an uncommon and unique clinical phenomenon that occurs in a minor proportion of serious infectious diseases. SPE is a nonthrombotic, pathogen-containing thrombus causing bacterial embolism in the pulmonary vasculature derived from a primary infection site via venous circulation. During the last 2-3 decades, the epidemiology of SPE has changed due to the increasing incidence of extrapulmonary sources of infection $(1,2)$. Klebsiella pneumoniae (K. pneumonia) is becoming the leading causative pathogen in gram-negative bacteria, and is only secondary to Staphylococcus aureus, the most common pathogenic bacterium leading to SPE (2-4). The diagnosis of SPE mainly depends on clinical evidence of infection with definite microorganism culture results and radiologic findings. The typical computed tomography (CT) scan findings that indicate SPE include peripheral nodules with or without cavities, wedge-shaped peripheral lesions near the visceral pleura or inter-lobar pleura, and pleural effusion (3).

In recent years, along with numerous populationbased studies from other Asian areas, hyper-virulent $K$. pneumoniae (HvK.P) has emerged in Chinese societies (4-6); K. pneumoniae-associated infection has been encountered with increasing frequency in emergency departments in Shanghai, China; and HvK.P causes rapidonset fatal infection that is prone to invade the immunecompromised subjects. As an infectious condition with presentations varying from common respiratory syndromes to severe sepsis, $K$. pneumoniae infection and its complications continue to garner the interest of clinical researchers. Of note, a minority of patients with primary extra-pulmonary $K$. pneumoniae have developed SPE as a more severe phenotype with a higher medical burden, puzzling clinicians $(2-4,6)$. However, SPE has not been well regarded in the existing guidelines for sepsis, and a limited SPE-K. pneumonia spectrum has been described in China.

Our emergency department has accepted numerous patients with $K$. pneumoniae infection and provided them with a timely diagnosis and effective interventions. We thus conducted a retrospective study of K. pneumoniaeinfected cases with primarily extrapulmonary infection, such as hematogenous infection and liver abscess (KPLA), who were treated in our emergency department from 2014 to 2019 and focused on the phenomenon of SPE to help clinicians improve the early suspicion and recognition of this potentially life-threatening condition and strengthen the clinical experience.
We present the following article in accordance with the STROBE Reporting Checklist (available at http://dx.doi. org/10.21037/apm-19-648).

\section{Methods}

\section{Patients and clinical data}

This retrospective study was conducted in the emergency department of Ren Ji Hospital, a tertiary referral teaching hospital affiliated with Shanghai Jiao Tong University School of Medicine. Records of patients who were consecutively admitted to the emergency ward and emergency intensive care unit (EICU) with an episode of extrapulmonary $K$. pneumoniae-SPE were retrieved between January 2014 and December 2019. Patient data were collected from the hospital information management system Ver. 5, a laboratory database of microorganisms and a radiological database. The medical records were reviewed and analyzed, including patients' demographic variables, clinical presentations, underlying conditions or relevant comorbidities, primary source of infected organ, laboratory data, microbiologic culture results with antimicrobial susceptibilities, radiological findings, treatment, complications, and clinical outcomes. The information and health-care identification numbers of the patients were encrypted.

\section{Imaging modality}

The high-resolution CT (HRCT) scanning (GE Discovery, HD 750, USA) parameters were as follows: $120 \mathrm{kV}, 100$ to $320 \mathrm{~mA}, 0.8$-second rotation time, $1 \mathrm{~mm}$ slice thickness, and $1 \mathrm{~mm}$ interval. The images were reviewed at a lung window setting level of 700 Hounsfield units (HU) and width of $1600 \mathrm{HU}$ and a mediastinum window setting level of $40 \mathrm{HU}$ and width of $400 \mathrm{HU}$. At least two radiologists independently evaluated the thoracic CT findings. Groundglass nodule (GGN) was defined as a hazy increase in attenuation without obscuration of vascular markings, and focal consolidation was defined as a localized increase in lung attenuation with obscuration of vascular markings (7).

\section{Definitions for critical conditions}

Acute respiratory failure was defined as an arterial partial pressure of oxygen $<60 \mathrm{mmHg}$ or an arterial partial pressure of carbon dioxide $\left(\mathrm{PaCO}_{2}\right)>45 \mathrm{mmHg}$ to produce respiratory acidosis $(\mathrm{pH}<7.35)$ while breathing air at normal atmospheric pressure $(8,9)$. The diagnostic criterion 
for acute kidney injury (AKI) was an increase in serum creatinine by at least $0.3 \mathrm{mg} / \mathrm{dL}$ within 48 hours (10). The Berlin definition of acute respiratory distress syndrome (ARDS) was employed (11).

\section{Diagnostic criteria for SPE}

The inclusion criteria for SPE diagnosis were adopted as follows: (I) the thoracic HRCT findings included the following patterns: peripheral pleural based wedge-shaped opacity or interlobular fissure, a feeding vessel sign, multiple nodular lesions with or without cavities, patchy groundglass opacities, multifocal lung infiltrates, air bronchograms within a nodule, focal consolidations, lung abscesses, halo signs, and pleural effusion $(1,12,13)$; (II) the presence of a primary extrapulmonary source of $K$. pneumoniae infection as a potential hematogenous source and documented bacteremia; (III) existing septic thrombophlebitis defined as acute venous thrombosis evidenced by ultrasound and/or CT scan; and (IV) clinical improvement and radiographic resolution after appropriate antibiotic therapy.

The exclusion criteria were as follows: (I) other potential explanations of lung lesions, including patients with other diagnoses of tuberculosis, invasive pulmonary fungal infection, possible lung tumor primary or metastasis; (II) patients with insufficient medical records $(1,12,13)$.

The protocol for the research project and human study was approved by the Ethics Committee of Ren Ji Hospital (No.[2016]080k), and the study was performed in accordance with the ethical standards outlined in the 2013 version of the Declaration of Helsinki. Because of the retrospective nature of the research, the requirement for informed consent was waived.

Categorical variables were presented as a number (\%). All continuous variables were presented as the mean \pm standard deviation (SD) or as the median (IQR) for data with a normal distribution and homogeneity of variance.

\section{Results}

\section{Demographic data}

A total of 110 patients with extrapulmonary infection of $K$. pneumoniae were included, among which 33 patients with typical clinical features and abnormal chest HRCT scan findings met the inclusion criteria of SPE (19 males, $57.8 \%$ ), with a mean age of $61.03 \pm 13.95$ years. The average duration of hospitalization was $26.88 \pm 17.76$ days (Table 1 ).

\section{Clinical features, pathogenic microbe, comorbidities}

The clinical manifestations included a febrile and generally fragile state $(100 \%)$ and respiratory symptoms $(n=6,18.2 \%)$, most commonly cough/sputum, dyspnea, and hemoptysis. Symptoms of the digestive system $(n=13,39.4 \%)$ included epigastric discomfort, anorexia/vomiting, and diarrhea. There were 9 patients required intensive care, among whom 8 (24.2\%) developed septic shock and $2(6.0 \%)$ experienced acute respiratory failure. Severe complication of endophthalmitis occurred in 2 patients (6.0\%) (Table 1). One patient was died of septic shock and multiple organ dysfunction syndrome (MODS) after accepting the resection of perirectal abscess, who had long-term medical history of uncontrolled primary Sjögren's syndrome.

All K. pneumonia isolates were susceptible to cephalosporines, aminoglycosides, or levofloxacin, and susceptibility to ampicillin/sulbactam, piperacillin, and carbapenems varied. No nosocomial or multi-resistant strains were identified.

The most common source of infection of the 33 cases was pyogenic liver abscess $(n=26,78.8 \%)$, followed by haematogenous infection $(n=8,24.2 \%)$, intestinal infection $(n=3,9.1 \%)$, and ascites infection $(n=1,3.0 \%)$. Thrombophlebitis or venous thrombosis occurred in 4 different individuals $(12.1 \%), 2$ cases $(6.0 \%)$ with lower limb thrombophlebitis (1 was in popliteal vein), 1 case was inferior vena cava thrombosis, and thrombogenesis involved branch of the portal vein observed in other one patient. Coexistence liver abscess and bacteremia was observed in 3 patients (9.1\%). Endogenous endophthalmitis was found in 2 cases $(6.0 \%)$, among which 1 patient had concurrence of liver abscess, bacteremia and endophthalmitis, another patient had bacteremia, lower limb thrombophlebitis and endophthalmitis simultaneously (Tables 1,2).

Underlying diseases or comorbidities included diabetes mellitus ( $\mathrm{n}=24,72.7 \%)$, hypertension $(\mathrm{n}=11,33.3 \%)$, cerebrovascular disease $(n=5,15.1 \%)$, cholelithiasis or biliary tract disease $(n=4,12.1 \%)$, hyperthyroidism $(n=1$, $3.0 \%)$, chronic hepatitis $(\mathrm{n}=2,6.0 \%)$, and chronic renal insufficiency $(\mathrm{n}=3,9.1 \%)$ (Table 1).

\section{Imaging findings}

The HRCT manifestations of the SPE cases included the following: peripheral wedge-shaped opacity $(n=12$, $36.4 \%)$, a feeding vessel sign $(\mathrm{n}=3,9.1 \%)$, multiple nodular lesions with or without cavities $(\mathrm{n}=5,15.2 \%)$, 
Table 1 Clinical characteristics of the 33 SPE cases with $K$. Pneumoniae infection

\begin{tabular}{|c|c|}
\hline Clinical characteristics & Value \\
\hline Age (year) & $61.03 \pm 13.95$ \\
\hline Sex (male), n (\%) & $19(57.8 \%)$ \\
\hline Hospital duration (day) & $26.88 \pm 17.76$ \\
\hline \multicolumn{2}{|l|}{ Presented symptoms, n (\%) } \\
\hline Fever & $33(100 \%)$ \\
\hline General weakness & $11(33.3 \%)$ \\
\hline Temperature peak in hospital $\left({ }^{\circ} \mathrm{C}\right)$ & $39.21 \pm 0.54$ \\
\hline Cough/sputum & $3(9.1 \%)$ \\
\hline Shortness of breath & $5(15.1 \%)$ \\
\hline Altered mental status & $4(12.1 \%)$ \\
\hline Hemoptysis & $1(3.0 \%)$ \\
\hline Right upper quadrant abdominal pain & $5(15.1 \%)$ \\
\hline Diarrhea & $3(9.1 \%)$ \\
\hline Anorexia/vomiting & $5(15.1 \%)$ \\
\hline \multicolumn{2}{|l|}{ Comorbidities, n (\%) } \\
\hline Diabetes mellitus & $24(72.7 \%)$ \\
\hline Hypertension & $11(33.3 \%)$ \\
\hline Cerebrovascular disease & $5(15.1 \%)$ \\
\hline Cholecystolithiasis & $4(12.1 \%)$ \\
\hline Hyperthyroidism & $1(3.0 \%)$ \\
\hline Chronic hepatitis & $2(6.0 \%)$ \\
\hline Chronic renal insufficiency & $3(9.1 \%)$ \\
\hline \multicolumn{2}{|l|}{ Complications, n (\%) } \\
\hline Septic shock & $8(24.2 \%)$ \\
\hline Acute respiratory failure & $2(6.0 \%)$ \\
\hline Acute kidney injury & $7(21.2 \%)$ \\
\hline Liver function impairment & $10(30.3 \%)$ \\
\hline Endogenous endophthalmitis & $2(6.0 \%)$ \\
\hline \multicolumn{2}{|l|}{ Source of infection, n (\%) } \\
\hline Liver abscess & $26(78.8 \%)$ \\
\hline Haematogenous infection & $8(24.2 \%)$ \\
\hline Intestinal infection & $3(9.1 \%)$ \\
\hline Ascites infection & $1(3.0 \%)$ \\
\hline \multicolumn{2}{|l|}{ Treatment, n (\%) } \\
\hline Antibiotics & $33(100 \%)$ \\
\hline Puncture drainage of hepatic abscess & $21(63.6 \%)$ \\
\hline Ophthalmectomy & $1(3.0 \%)$ \\
\hline
\end{tabular}

The data were presented as mean \pm SD. SPE, septic pulmonary embolism.
Table 2 The imaging findings of 33 SPE patients with $K$. Pneumoniae infection

\begin{tabular}{lc}
\hline Chest imaging findings & $\mathrm{N}(\%)$ \\
\hline Peripheral wedge-shaped opacity & $12(36.4)$ \\
Feeding vessel sign & $3(9.1)$ \\
Multiple nodular lesions with or without cavities & $5(15.2)$ \\
Multifocal lung ill-infiltrations & $15(45.5)$ \\
Patchy ground-glass opacities & $6(18.2)$ \\
Focal consolidations & $9(27.3)$ \\
Lung abscess or air bronchograms within a nodule & $4(12.1)$ \\
Pleural effusion & $21(63.6)$ \\
Other imaging findings & \\
Thrombophlebitis/venous thrombus & $4(12.1)$ \\
Endogenous endophthalmitis & $2(6.0)$ \\
\hline
\end{tabular}

SPE, septic pulmonary embolism.

multifocal lung ill-infiltrations $(\mathrm{n}=15,45.5 \%)$, patchy ground-glass opacities $(\mathrm{n}=6,18.2 \%)$, focal consolidations $(\mathrm{n}=9,27.3 \%)$, lung abscesses $(\mathrm{n}=4,12.1 \%)$, and pleural effusion ( $\mathrm{n}=21,63.6 \%$ ) (Table 2, Figures 1-3). Thrombogenesis involved branch of the portal vein was observed in 1 patient with pyogenic liver abscess (Figure 2D). One patient had bacteremia and endophthalmitis accepted eyeball B-ultrasound detection (Figure 3C). All the patients underwent an echocardiogram, and no mitral or tricuspid valve infective endocarditis was found.

\section{Treatment, outcomes}

All the 33 patients with SPE received broad-spectrum antibiotics in the initial time, followed by guidance from the results of culture and susceptibility targeted pathogen of K. pneumonia. There were 21 patients $(63.6 \%)$ received ultrasound-guided puncture drainage, 1 patient with unilateral endophthalmitis accepted ophthalmectomy, all the patients were survived after appropriate treatment. In total, reexamination of thoracic HRCT was conducted in 7 patients after treatment, and imaging improvement after treatment was demonstrated (Figure 1).

\section{Discussion}

In the present study, we describe the spectrum of SPE in subjects with extrapulmonary infection of $K$. pneumonia 

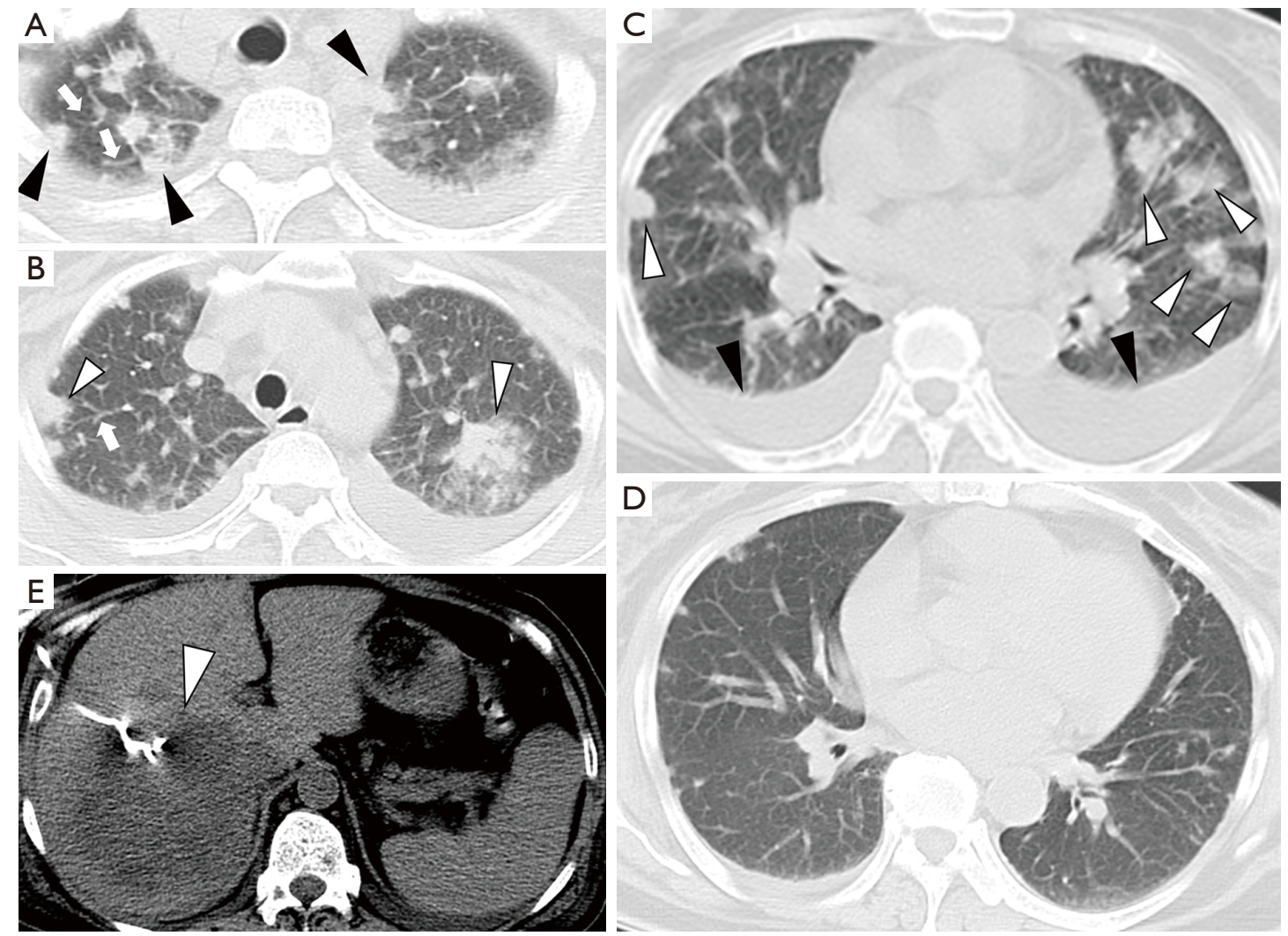

Figure 1 Pulmonary high-resolution computed tomography (HRCT) findings of invasive Klebsiella pneumoniae liver abscess (KPLA) in a 56-year-old diabetic woman who complained of repeated fever, fatigue, nausea, and cough for more than 20 days before admission to the hospital. (A,B,C) The cross-sectional HRCT scan showed bilateral lungs filled with multiple nodules, clump-shaped masses, groundglass opacities, and pleural effusion. There were multiple peripheral sub-pleural, wedge-shaped opacities (arrowheads) and peripheral nodules with feeding vessels (arrows). (D) After treatment for two weeks, the lung window of HRCT showed significant regression of the multiple scattered pulmonary foci above, and pleural effusion decreased significantly. (E) The abdominal CT scan of this patient displayed a hypodense abscess located in the right hepatic lobe with a drainage tube in the cavity.

admitted to our emergency ward and EICU, mostly pyogenic liver abscess (KPLA) and bloodstream infection. $K$. pneumonia-SPE was identified in 33 of 110 patients (27.3\%), which is some higher than the rate reported by previous studies $(4.5 \%$ and $6 \%)(12,14)$, most likely because chest HRCT scans were performed on all the patients as a routine examination to avoid missed diagnosis.

The Asian area has a high prevalence of communityacquired $K$. pneumonia infection, including peripheral endogenous and exogenous site (mainly KPLA) caused by $H v K . P$. The lung is the target organ that is liable to be involved with metastatic infection (4,15-22). SPE usually presents as consolidations, multiple nodules with or without cavities and GGN in CT images $(1,2,13,22)$; these features bear more similarities to those of pneumonia, which may confuse clinicians in the initial or progressive stage of the disease. Although a rare case study obtained pathologic findings of SPE described as many neutrophils and foamy histocytes (23), percutaneous needle aspiration biopsy is not easily available in clinical practice, and the diagnosis of SPE is thus mainly dependent on clinical and radiological judgement. Therefore, it is challenging for emergency physicians to distinguish SPE from pneumonia through imaging along with clinical features.

Extrapulmonary $K$. pneumoniae infection and the rapid aftermath of SPE indicated that K. pneumoniae-associated SPE can occur potentially catastrophically without early intervention. The main prevalent symptoms are fever and dyspnea. However, compared to those of thrombotic pulmonary embolism or sepsis-induced acute respiratory 

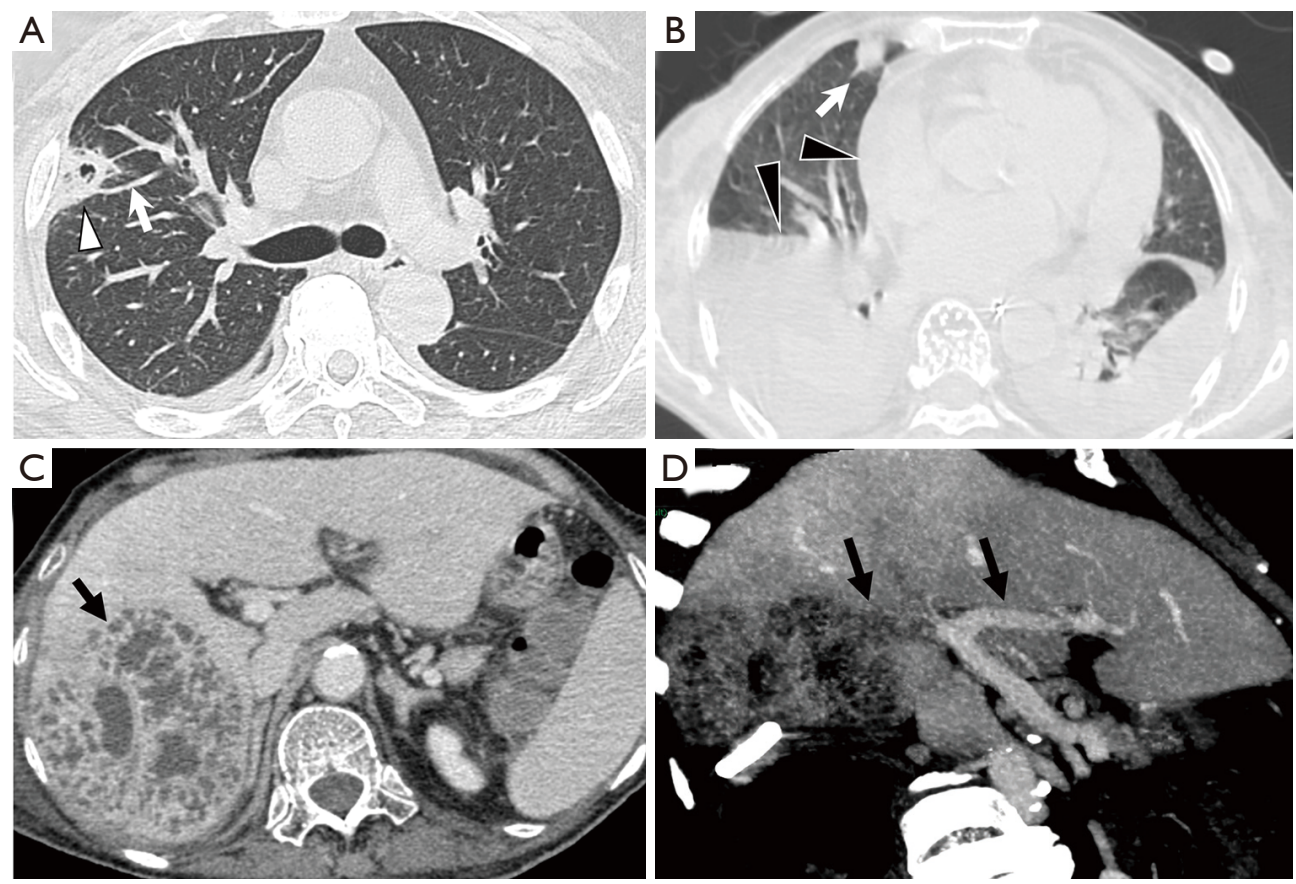

Figure 2 Pyogenic liver abscess with septic pulmonary embolism in a 66-year-old man (A) and a 77-year-old woman (B-D) with poorly controlled diabetes, both of whom were positive for Klebsiella pneumoniae, detected from aspirated pus and blood cultures. (A) The crosssectional scan of HRCT at the early stage of the disease showed a peripheral pleural-based, wedge-shaped nodule with cavitation inside (white arrowhead), accompanied by a feeding vessel sign (white arrow). (B) The cross-sectional HRCT scan of the lung window showed bilateral plural effusion and pericardial effusion (black arrowheads). There was a peripheral wedge-shaped nodule close to the adjacent pleura (white arrow). (C) The enhanced abdominal CT scan identified a large hypodense and multiseptated abscess involving the right hepatic lobe; (D) the right branch of the portal vein was missing, which was suggestive of right portal vein embolism, and the accompanying phenomenon was hypo-perfusion in the corresponding blood supply area of the liver parenchyma (black arrows).

distress syndrome (ARDS), the lesions of SPE were shown to be reversible after aggressive treatment, such as broadspectrum or microbiological test-guided antibiotic therapy or early treatment of empyema by applying liver abscess drainage to deduce the occurrences of lethal conditions, in most of the patients $(1,3,13,14,21)$. This phenomenon also existed in our study and could explain why only 1 patient developed acute respiratory failure, and persistent respiratory failure was not common due to timely control of the primary infection and advanced life support. We observed obvious regression of pulmonary infiltrates or nodules in 7 KPLA patients who underwent repeated HRCT scans after antiseptic treatment (Figure 1).

As another important phenotype of SPE, thrombophlebitis or venous thrombosis were observed in four different cases. Septic lower limb thrombophlebitis was identified in 2 cases; right portal vein embolism in an old female KPLA patient was detected by abdominal enhanced
CT scan (Figure 2); inferior vena cava thrombosis in another old female patient with KPLA; Among the cases of hematogenous spread, the complex clinical manifestations of SPE, combined with lower limb thrombophlebitis and endogenous endo-ophthalmitis in a middle-aged diabetic man whose blood culture was positive for K. pneumoniae, revealed the rare and severe phenotypes of this type of crippling disease (Figure 3).

Importantly, it is well known that underlying or comorbid diseases, as well as immunocompromised conditions, contribute to the occurrence of SPE (22-25). For example, an old male patient with stage 5 chronic kidney dysfunction (CKD 5) identified as having hematogenous and ascetic $K$. pneumoniae infection showed characteristic cavitary nodules, ill-defined lung infiltration, and focal consolidation on the pulmonary HRCT scan during the disease progress. Additionally, in the 33 patients with $K$. pneumonia-SPE, $24(72.7 \%)$ had diabetes with a high value of glycosylated 

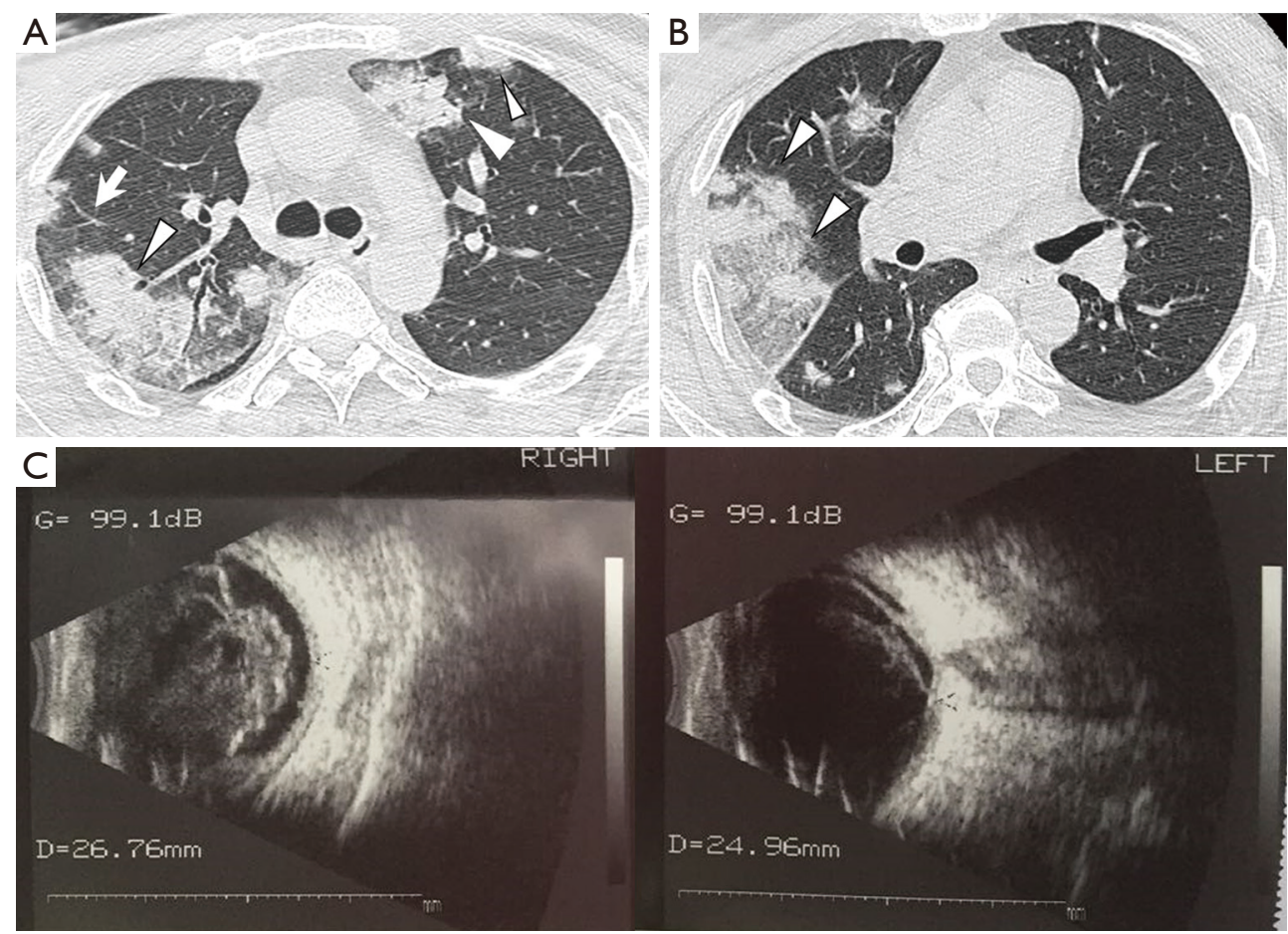

Figure 3 A rare and severe case of Klebsiella pneumoniae complicated with septic pulmonary emboli, septic thrombophlebitis, and endophthalmitis. A 45-year-old diabetic man who manifested with high fever, cough, and right lower limb swelling, along with visual deterioration, visited the emergency room. His blood culture was positive and identified as a subtype of Klebsiella pneumoniae. (A,B) Lung windows of cross-sectional HRCT scan showed multiple patchy ground-glass and nodular opacities in the left and right lungs, several peripheral nodules abutting the adjacent pleura (white arrowheads) and peripheral nodules with feeding vessels (arrow). (C) Eyeball B-scan ultrasonography of this patient. There were large chunks of turbidity in the right vitreous body, and retinal detachment was suspicious, indicating severe endophthalmitis in the right eye.

hemoglobin (HbA1c), indicating that poor glycemic control may have a close relationship with septic metastatic infection.

Notably, the phenomena that new SPE continued to develop regardless of aggressive antibiotic therapy and that pigtail catheter drainage of the liver abscess was also observed in our study indicated that SPE may be sequential, inevitable and reversible $(12,14,21,22,26)$. That is, chest HRCT should be performed in all patients with the isolation of bacteria from the bloodstream and definite infectious focus (mainly in KP-PLA), especially those with SPE risk factors such as those described above. Furthermore, in a proportion of cases, follow-up CT scans showing the regression or disappearance of lung lesions reaffirmed the diagnosis of SPE (Figure 1C,D).

Fortunately, in this study, the pattern of antimicrobial susceptibility indicated that the K. pneumonia strains were community acquired and were not naturally multi-resistant strains. It is also worth mentioning that no cardiac SPE, which was excluded by echocardiographic measurement, was found in this study. The possible reasons were as follows: (I) the most common pathogenic bacteria of cardiac SPE is Staphylococcus aureus rather than K. pneumoniae. (II) No patient had a history of intravenous drug abuse, cardiac device implants, congenital heart disease, or tricuspid valve infective endocarditis, as historically documented from other countries $(25,27)$.

\section{Limitations}

Two limitations were encountered when conducting this study. First, data were collected retrospectively from a single medical tertiary center, which may have resulted in selection bias. Second, histopathological confirmation was unavailable, and the current SPE diagnosis was mainly based on CT findings and clinical evidence of infection with 
a definitive bacteriological result.

\section{Conclusions}

The main findings of this study are as follows. First, clinical characteristic and unique imaging signs of SPE in patients with sepsis who require critical care, especially those with vital organ involved and pyogenic liver abscess, should be provided. Second, in patients with $K$. pneumonia infection, emergency physicians should be aware of SPEs so they can distinguish SPE from common pneumonia and promptly initiate treatment. It is important to pay more attention to its different terrible appearances in the emergency department, where the patients always obtain first aid and further treatment. Misdiagnosis and treatment will lead to serious consequences. Third, early identification, appropriate antibiotic therapy, surgical intervention and respiratory support are essential for the treatment of patients with SPE who require critical care.

\section{Acknowledgments}

We thank the patients for their participation in this study; and Dr. Chen'an Ye, Dr. Ting Gao, and Dr. Zhu'ang Ni from the Department of Emergency Medicine, Ren Ji Hospital, for their medical and technical assistance. We thank all the respective reviewers and editors who provide valuable input to each submission.

Funding: This project was partially supported by grants from the "Science and Technology Commission of Shanghai Municipality” (Grants nos. 2015DZ1941307).

\section{Footnote}

Reporting Checklist: The authors have completed the STROBE Reporting Checklist. Available at http://dx.doi. org/10.21037/apm-19-648

Data Sharing Statement: Available at http://dx.doi. org/10.21037/apm-19-648

Conflicts of Interest: All authors have completed the ICMJE uniform disclosure form (available at http://dx.doi. org/10.21037/apm-19-648). The authors have no conflicts of interest to declare.

Ethical Statement: The authors are accountable for all aspects of the work in ensuring that questions related to the accuracy or integrity of any part of the work are appropriately investigated and resolved. The protocol for the research project and human study was approved by the Ethics Committee of Ren Ji Hospital (No.[2016]080k), and the study was performed in accordance with the ethical standards outlined in the 2013 version of the Declaration of Helsinki. Because of the retrospective nature of the research, the requirement for informed consent was waived.

Open Access Statement: This is an Open Access article distributed in accordance with the Creative Commons Attribution-NonCommercial-NoDerivs 4.0 International License (CC BY-NC-ND 4.0), which permits the noncommercial replication and distribution of the article with the strict proviso that no changes or edits are made and the original work is properly cited (including links to both the formal publication through the relevant DOI and the license). See: https://creativecommons.org/licenses/by-nc-nd/4.0/.

\section{References}

1. Cook RJ, Ashton RW, Aughenbaugh GL, et al. Septic pulmonary embolism: presenting features and clinical course of 14 patients. Chest 2005;128:162-6.

2. Brenes JA, Goswami U, Williams DN. The association of septic thrombophlebitis with septic pulmonary embolism in adults. Open Respir Med J 2012;6:14-9.

3. Goswami U, Brenes JA, Punjabi GV, et al. Associations and outcomes of septic pulmonary embolism. Open Respir Med J 2014;8:28-33.

4. Ye M, Tu J, Jiang J, et al. Clinical and Genomic Analysis of Liver Abscess-Causing Klebsiella pneumoniae Identifies New Liver Abscess-Associated Virulence Genes. Front Cell Infect Microbiol 2016;6:165.

5. Zhang Y, Sun J, Mi C, et al. First report of two rapid-onset fatal infections caused by a newly emerging hypervirulent

K. Pneumonia ST86 strain of serotype K2 in China. Front Microbiol 2015;6:721.

6. Liu L, Chen W, Lu X, et al. Pyogenic Liver Abscess: A Retrospective Study of 105 Cases in an Emergency Department from East China. J Emerg Med 2017;52:40916.

7. Franquet T, Herold CJ. Community-acquired pneumonia. In: Müller NL, Silva CI. 1st ed. Imaging of the chest. Philadelphia: Saunders/Elseviersemi, 2008:290-321.

8. Knaus WA, Draper EA, Wagner DP, et al. APACHE II: a severity of disease classification system. Crit Care Med 1985;13:818-29. 
9. Ferreira FL, Bota DP, Bross A, et al. Serial evaluation of the SOFA score to predict outcome in critically ill patients. JAMA 2001;286:1754-8.

10. Mehta RL, Kellum JA, Shah SV, et al. Acute Kidney Injury Network: report of an initiative to improve outcomes in acute kidney injury. Crit Care 2007;11:R31.

11. Ferguson ND, Fan E, Camporota L, et al. The Berlindefinition of ARDS: an expanded rationale, justification, and supplementary material. Intensive Care Med 2012;38:1573-82.

12. Chou DW, Wu SL, Chung KM, et al. Septic pulmonary embolism caused by a Klebsiella pneumoniae liver abscess: clinical characteristics, imaging findings, and clinical courses. Clinics (Sao Paulo) 2015;70:400-7.

13. Chou DW, Wu SL, Chung KM, et al. Septic Pulmonary Embolism Requiring Critical Care: Clinicoradiological Spectrum, Causative Pathogens and Outcomes. Clinics (Sao Paulo) 2016;71:562-9.

14. Lee SS, Chen YS, Tsai HC, et al. Predictors of septic metastatic infection and mortality among patients with Klebsiella pneumoniae liver abscess. Clin Infect Dis 2008;47:642-50.

15. Lee KH, Hui KP, Tan WC, et al. Klebsiella bacteremia: a report of 101 cases from National University Hospital, Singapore. J Hosp Infect 1994;27:299-305.

16. Tsai FC, Huang YT, Chang LY, et al. Pyogenic liver abscess as endemic disease, Taiwan. Emerg Infect Dis 2008;14:1592-600.

17. Zhu X, Wang S, Jacob R, et al. 10-year retrospective analysis of clinical profiles, laboratory characteristics and management of pyogenic liver abscesses in a Chinese hospital. Gut Liver 2011;5:221-7.

18. Mangukiya DO, Darshan JR, Kanani VK, et al. A prospective series case study of pyogenic liver abscess: recent trends in etiology and management. Indian J Surg
2012;74:385-90.

19. Liu Y, Wang JY, Jiang W. An increasing prominent disease of Klebsiella pneumoniae liver abscess: etiology, diagnosis, treatment. Gastroenterol Res Pract 2013;2013:258514.

20. Ghosh S, Sharma S, Gadpayle AK, et al. Clinical, laboratory, and management profile in patients of liver abscess from northern India. J Trop Med 2014;2014:142382.

21. Yao Z, Zheng J, Si Y, et al. Pneumocardia and Septic Pulmonary Embolism Due to Nongas-Forming Liver Abscess: A Case Report. Medicine (Baltimore) 2018;97:e13096.

22. Ojeda Gómez JSA, Carrillo Bayona JA, Morales Cifuentes LC. Septic Pulmonary Embolism Secondary to Klebsiella pneumoniae Epididymitis: Case Report and Literature Review. Case Rep Radiol 2019;2019:5395090.

23. Lee SJ, Weon YC, Cha HJ, et al. A case of atypical skull base osteomyelitis with septic pulmonary embolism. J Korean Med Sci 2011;26:962-5.

24. Zheng B, Dai Y, Liu Y, et al. Molecular Epidemiology and Risk Factors of Carbapenem-Resistant Klebsiella pneumoniae Infections in Eastern China. Front Microbiol 2017;8:1061.

25. Yasar KK, Pehlivanoglu F, Gursoy S, et al. Tricuspid Endocarditis and Septic Pulmonary Embolism in an Intravenous Drug User with advanced HIV Infection. Oman Med J 2011;26:365-7.

26. Takeda K, Tanaka K, Kumamoto T, et al. Septic pulmonary embolism originated from subcutaneous abscess after living donor liver transplantation: a pitfall of postoperative management. Clin J Gastroenterol 2013;6:378-2.

27. Song XY, Li S, Cao J, et al. Cardiac septic pulmonary embolism: A retrospective analysis of 20 cases in a Chinese population. Medicine (Baltimore) 2016;95:e3846.
Cite this article as: Zhang X, Yang Q, Gao B, Wang J, Tian L, Hua J, Zhu C, Lu X. Klebsiella pneumoniae infection associated septic pulmonary embolism in an emergency department from east China. Ann Palliat Med 2021;10(2):1521-1529. doi: 10.21037/ apm-19-648 